\title{
Autoantibodies in systemic vasculitis
}

\author{
Alexandre Wagner Silva de Souza* \\ Rheumatology Division, Department of Internal Medicine, Universidade Federal de São Paulo-Escola Paulista de Medicina, \\ São Paulo, Brazil
}

Keywords: autoantibodies, systemic vasculitis, ANCA, cryoglobulinemia, anti-GBM antibodies

\section{Introduction}

Systemic vasculitis is a heterogeneous group of disorders characterized by inflammation and necrosis in the vessel wall. The diagnosis of a systemic vasculitis is challenging, because patients usually present a broad spectrum of manifestations that vary according to the predominant size of vessels affected, organs and systems involved, and the extent of the inflammatory process (1). In systemic vasculitis, disease manifestations usually cluster into clinical phenotypes and definite diagnosis rely on confirmation by tissue biopsy, angiography, or by serologic tests. However, when a systemic vasculitis is under investigation, it is of paramount importance to keep in mind vasculitis mimics (e.g., genetic vascular disorders and atheroembolic diseases) and secondary causes (i.e., infections, malignancy, connective tissue disorders, or drugs) (2).

The main serologic tests for the diagnosis of primary systemic vasculitides are antineutrophil cytoplasmic antibodies (ANCA), cryoglobulins, anti-glomerular basement membrane (anti-GBM) antibodies, and anti-C1q antibodies (3). Although, several other autoantibodies have been investigated in systemic vasculitis, the clinical usefulness of these antibodies still needs further investigation $(1,2)$ (Table 1). Herein, the main autoantibodies and their clinical associations in systemic vasculitis are reviewed.

\section{OPEN ACCESS}

Edited by:

Edward K. L. Chan, University of Florida, USA

Reviewed by:

Elena Csernok,

University of Lübeck, Germany Roger A. Levy, Universidade do Estado do Rio de Janeiro, Brazil

*Correspondence:

Alexandre Wagner Silva de Souza alexandre_wagner@uol.com.br

Specialty section:

This article was submitted to B Cell Biology, a section of the journal Frontiers in Immunology

Received: 31 January 2015 Accepted: 03 April 2015 Published: 22 April 2015

Citation:

Silva de Souza AW (2015) Autoantibodies in systemic vasculitis.

Front. Immunol. 6:184. doi: 10.3389/fimmu.2015.00184

\section{Antineutrophil Cytoplasmic Antibodies}

Antineutrophil cytoplasmic antibodies are the diagnostic biomarkers for some small-vessel necrotizing vasculitis such as granulomatosis with polyangiitis (GPA) (formerly Wegener's granulomatosis), mycroscopic polyangiitis (MPA), eosinophilic granulomatosis with polyangiitis (EGPA) (formerly Churg-Strauss syndrome), and renal limited vasculitis (RLV). The main target antigens of ANCA are proteinase 3 (PR3) and myeloperoxidase (MPO) located on primary granules of neutrophils and lysosomes of monocytes. ANCA reactivity is variable and differences in affinity of ANCA with PR3 antigen have been described with a higher inhibition of PR3 activity observed in GPA patients in remission compared with those with active disease. Unlike, PR3-ANCA, myeloperoxidaseantineutrophil cytoplasmic antibodies (MPO-ANCA) recognize a restricted number of epitopes in MPO and do not impair its enzymatic activity. When ANCA bind to their target antigen, they activate neutrophils and induce the release of granule enzymes, pro-inflammatory cytokines, and the generation of respiratory burst, leading to endothelial damage and eventually to the vasculitic process (4).

Currently, the main assays for the detection of ANCA in sera are indirect immunofluorescence (IIF) on ethanol-fixed human neutrophils and enzyme-linked immunosorbent assay (ELISA) for antigen specificity. The International Consensus Statement on Testing and Reporting ANCA recommends the combination of both techniques (IIF and ELISA) for the detection of ANCA in patients under investigation for ANCA-associated vasculitis, since $10 \%$ of ANCA positive GPA or MPA patients present only IIF positive results (5). The main patterns observed in IIF are the cytoplasmic (C-ANCA) pattern that displays a diffuse granular cytoplasmic staining with interlobular accentuation and the perinuclear (P-ANCA) pattern that shows perinuclear fluorescence with nuclear extension. C-ANCA is mostly associated with anti-PR3 antibodies, whereas 
TABLE 1 | Autoantibodies in primary systemic vasculitides

\begin{tabular}{ll}
\hline Vasculitis & Autoantibodies \\
\hline Takayasu arteritis & AECA \\
& Anti-aorta antibodies \\
& Anti-ferritin antibodies \\
& Anti-annexin $V$ antibodies \\
& Anti-monocyte antibodies \\
Giant cell arteritis & AECA \\
Polyarteritis nodosa & Anti-ferritin antibodies \\
Kawasaki disease & AECA \\
ANCA-associated vasculitis & AECA \\
& AECA \\
Immune complex vasculitis & MPO-ANCA \\
& Anti-LAMP-2 antibodies \\
& Anti-C1q antibodies \\
& Anti-GBM antibodies \\
Cryoglobulins \\
IgA-ANCA \\
Rheumatoid factor \\
\end{tabular}

AECA, antiendothelial cell antibodies; ANCA, antineutrophil cytoplasmic antibodies; GBM, glomerular basement membrane; LAMP-2, lysosome associated membrane protein-2; MPO, myeloperoxidase; $P R 3$, proteinase 3.

$\mathrm{P}-\mathrm{ANCA}$ is associated with anti-MPO antibodies. Another ANCA pattern described is the atypical ANCA, also referred as A-ANCA. A-ANCA usually shows perinuclear staining without nuclear extension, or diffuse flat cytoplasmic staining, or the combination of both cytoplasmic and nuclear/perinuclear staining on neutrophils $(5,6)$.

Antineutrophil cytoplasmic antibodies are positive in up to $90 \%$ of patients with active generalized GPA, while only $40 \%$ of patients with localized disease present a positive ANCA test. PR3-ANCA is found in up to $90 \%$ of GPA patients with a positive ANCA. The presence of MPO-ANCA is observed in MPA, EGPA, and with RLV with a rate of positivity of 70,50 , and $80 \%$, respectively. The combination of IIF and ELISA for PR3-ANCA or MPO-ANCA yields a specificity of $99 \%$ (4). ANCA specificity in ANCA-associated vasculitis is more closely related to disease phenotype rather than clinical diagnosis. Patients presenting renal involvement and small-vessel vasculitis without evidence of granulomatous inflammation are more likely to have MPO-ANCA, whereas PR3-ANCA is more associated with necrotizing granulomatous inflammation and more frequent relapses compared with MPO-ANCA. Moreover, results from a genome wide association study that evaluated HLA and SERPINA1 genes showed that genetic background in ANCA-associated vasculitis is related with ANCA-specificity rather than with disease phenotype. ANCA levels often fluctuate following disease activity, but they are not meant to guide therapeutic decisions (7).

Myeloperoxidase-antineutrophil cytoplasmic antibodies are also found in $30 \%$ of patients with anti-GBM disease (4). A-ANCA is associated with antibodies against other target antigens in cytoplasmic granules of neutrophils such as lactoferrin, lysozyme, azurocidin, elastase, cathepsin G, and bactericidal/permeability increasing enzyme (BPI) (8). A-ANCA may be found in inflammatory bowel disease, mostly ulcerative colitis, autoimmune hepatitis, primary sclerosing cholangitis, rheumatoid arthritis, and drug exposure or drug-induced ANCA-associated vasculitis $(6,8)$.
During the last few years, different immunoassays have been developed for the detection of ANCA including second and third generation ELISA tests, automated systems for analyzing fluorescence patterns, chemiluminescent immunoassays, lateral flow assay systems, and bead-based multiplex assays (6-8). The first generation ELISA used to detect PR3-ANCA and MPO-ANCA applies absorption coating methods with target antigens directly immobilized to the surface of the plate what induces masking and deformation of epitopes. This direct ELISA has significant variation amongst kits and lacks sensitivity $(6,7)$. Second and third generation ELISA tests were developed to improve sensitivity. The second generation ELISA is also referred as capture ELISA and uses capture molecules, mostly monoclonal antibodies to bind the target antigen to the plate surface, while the third generation ELISA uses anchor molecules to immobilize the antigen to the plate surface. A study that compared three generations of PR3-ANCA assays found the highest sensitivity with the third generation anchor ELISA: $96.0 \%$ [95\% confidence interval (CI): 79.6-99.3\%], followed by IIF: $92.0 \%$ (95\% CI: 73.9-98.8\%), by second generation capture ELISA: $72.0 \%(95 \%$ CI: $50.6-87.9 \%)$, and by first generation direct ELISA: $60.0 \%(95 \%$ CI: 38.7-78.8\%) (9).

Despite advances in assays to detect ANCA, to date the combination of IIF with the ELISA test to detect ANCA specificity is still recommended, preferably with second or third generation ELISA. Although, chemiluminescent immunoassay has similar or superior diagnosis performance for ANCA-associated vasculitis compared to conventional assays, the role of novel techniques to detect ANCA deserves further studies $(7,8)$.

\section{Anti-Lysosome Associated Membrane Protein-2 Antibodies}

The lysosome associated membrane protein-2 (LAMP-2) is a heavily glycosilated protein present in lysosomes, but it also traffics to the cell surface where it is expressed by monocytes, neutrophils, and endothelial cells. Moreover, LAMP-2 may be also found within the membrane of MPO/PR3 intracellular vesicles of neutrophils. Thus, anti-LAMP-2 antibodies are considered a subtype of ANCA. The pathogenicity of anti-LAMP-2 antibodies is highlighted by the striking $100 \%$ homology observed between the epitope $\mathrm{P}_{41-49}$ of LAMP-2 and a sequence in FimH, a bacterial adhesin present in fimbriated bacterial such as Escherichia coli (10).

Anti-LAMP-2 antibodies were firstly described in $87 \%$ of patients with RLV and this high prevalence of anti-LAMP-2 antibodies was further confirmed in a multicenter study that included patients with ANCA-associated vasculitis (i.e., GPA, MPA, and $\mathrm{RLV}$ ) at disease presentation from Austria, the Netherlands, and from the United Kingdom with a positivity of $89 \%, 91 \%$, and $80 \%$, respectively. In contrast, anti-LAMP-2 antibodies were detected in only $7 \%$ of patients in remission. However, the prevalence of anti-LAMP- 2 antibodies was only $21 \%$ in patients with ANCAassociated vasculitis from a study performed in North Carolina. The reasons for these controversial results are probably due to different methods used to detect anti-LAMP-2 antibodies in both studies and to the inclusion of patients in different phases of the disease (e.g., active and quiescent disease) in the latter study (10). 


\section{Cryoglobulins}

Cryoglobulins are typically immunoglobulins that precipitate in vitro at temperatures $<37^{\circ} \mathrm{C}$ and solubilize upon re-warming. Cryoglobulinemia refers to the presence of cryoglobulins in patient's serum. Besides the quantification of circulating cryoglobulins, it is also necessary to analyze the nature of circulating cryoglobulins (11). Brouet classification is the most frequent way of classifying cryoglobulins, this classification relies on the clonality of immunoglobulins and on rheumatoid factor activity. Type I cryoglobulinemia refers to the presence of monoclonal immunoglobulin, including IgM, IgG, IgA, or light chains (e.g., kappa or lambda) without rheumatoid factor activity. Type I cryoglobulinemia is usually associated with hematologic malignancies (e.g., Waldenström macroglobulinemia or multiple myeloma). Type II cryoglobulinemia is typically defined as the presence of polyclonal immunoglobulins associated with a monoclonal IgM with rheumatoid factor activity. Type III cryoglogulinemia is a mixture of polyclonal IgM and IgG. Both type II and III are referred as mixed cryoglobulinemia and are associated with chronic viral infections, mainly hepatitis $\mathrm{C}$ virus (HCV) and connective tissue diseases. In the latter group of diseases, cryoglobulins are a mixture of autoantibodies that become antigenic. The distinction between those autoantibodies and autoantigens is rather difficult. No other cause may be found in up to $10 \%$ of patients with mixed cryoglobulinemia and those cases are regarded as essential cryoglobulinemia (12).

Two clinical syndromes are recognized as caused by circulating cryoglobulins in serum: the hyperviscosity syndrome and cryoglobulinemic vasculitis. Although most patients with type I cryoglobulinemia are asymptomatic, the hyperviscosity syndrome is the main set of manifestations in this type of cryoglobulinemia and includes Raynaud's phenomenon, digital ischemia, renal failure, vision blurring, loss of vision, headache, vertigo, nystagmus, deafness, confusion, coma, and other neurological manifestations (12). Cryoglobulinemic vasculitis usually develops in patients with type II and III cryoglobulinemia and is manifested by palpable purpura, livedo reticularis, Raynaud's phenomenon, arthralgia, myalgias, glomerulonephritis, and peripheral neuropathy. In severe cases, patients present rapidly progressive glomerulonephritis, central nervous system vasculitis and/or pulmonary vasculitis (11).

\section{Anti-Glomerular Basement Membrane Antibodies}

Anti-glomerular basement membrane antibodies are biomarkers for the diagnosis of anti-GBM antibody disease (formerly Goodpasture's syndrome), which has been recently classified as immune complex small-vessel vasculitis affecting glomerular and/or pulmonary capillaries. Renal involvement is due to crescentic

\section{References}

1. Nataraja A, Mukhtyar C, Hellmich B, Langford C, Luqmani R. Outpatient assessment of systemic vasculitis. Best Pract Res Clin Rheumatol (2007) 21:713-32. doi:10.1016/j.berh.2007.01.004 glomerulonephritis and is usually manifested as rapidly progressive glomerulonephritis, whereas typical pulmonary involvement is pulmonary hemorrhage (3).

Anti-glomerular basement membrane antibodies may be detected by direct IF on renal biopsy, where a linear deposition of IgG is observed on glomerular capillaries. Alternatively, when renal biopsy cannot be performed the ELISA test is used to detect circulating anti-GBM antibodies in patients with active disease and its sensitivity ranges from 65 to $100 \%$. ELISA assays that use purified or recombinant alpha- 3 chain of collagen IV present the best sensitivity. Antigen specificity may also be confirmed by Western blot. IIF is rarely performed (13).

\section{Anti-C1q Antibodies}

Anti-C1q antibodies are biomarkers for the diagnosis of hypocomplementemic urticarial vasculitis (HUV). This entity has recently been classified as immune complex small-vessel vasculitis by 2012 Chapel Hill Consensus Conference. Common features of HUV include glomerulonephritis, cutaneous lesions (e.g., wheals that persist for more than $24 \mathrm{~h}$ ), arthralgia or arthritis, lung involvement, gastrointestinal vasculitis, and ocular inflammation (3).

\section{Other Autoantibodies}

Anti-endothelial cell antibodies (AECA) are a heterogeneous family of antibodies with multiple target antigens on endothelial cell membrane. AECA have been described in several primary systemic vasculitides such as Takayasu arteritis, giant cell arteritis, polyarteritis nodosa, GPA, MPA, EGPA, IgA vasculitis, Kawasaki disease, and Behçet's disease. However, the usefulness of AECA in clinical practice has still to be determined (14).

Anti-ferritin antibodies are novel IgG autoantibodies against human ferritin heavy chain and its terminal $\mathrm{N}$ peptide. Using the combination of results from different ELISA assays, anti-ferritin antibodies have been described in up to $92 \%$ of patients with giant cell arteritis/polymyalgia rheumatica and in $62 \%$ of Takayasu arteritis patients. However, anti-ferritin antibodies are also found in $28 \%$ of patients with systemic lupus erythematosus, in $22 \%$ of patients with febrile illnesses, and in $11 \%$ of patients with atherosclerotic disease, but not in healthy blood donors (15).

\section{Concluding Remarks}

To date, the investigation of circulating autoantibodies has been shown to be useful for diagnosis of systemic small vessel vasculitis including ANCA-associated vasculitis and immune complex vasculitis. No specific autoantibody has been shown to be of any help in diagnosis or in evaluating disease activity in patients with medium and large vessel vasculitis.

2. Jayne D. The diagnosis of vasculitis. Best Pract Res Clin Rheumatol (2009) 23:445-53. doi:10.1016/j.berh.2009.03.001

3. Jennette JC, Falk RJ, Bacon PA, Basu N, Cid MC, Ferrario F, et al. 2012 revised international chapel hill consensus conference nomenclature of vasculitides. Arthritis Rheum (2013) 65:1-11. doi:10.1002/art.37715 
4. Bosch X, Guilabert A, Font J. Antineutrophil cytoplasmic antibodies. Lancet (2006) 368:404-18. doi:10.1016/S0140-6736(06)69114-9

5. Savige J, Gillis D, Benson E, Davies D, Esnault V, Falk RJ, et al. International consensus statement on testing and reporting of antineutrophil cytoplasmic antibodies (ANCA). Am J Clin Pathol (1999) 111:507-13.

6. Csernok E, Moosig F. Current and emerging techniques for ANCA detection in vasculitis. Nat Rev Rheumatol (2014) 10:494-501. doi:10.1038/nrrheum. 2014.78

7. Sinico RA, Radice A. Antineutrophil cytoplasmic antibodies (ANCA) testing: detection methods and clinical application. Clin Exp Rheumatol (2014) 32(3 Suppl 82):S112-7.

8. Schulte-Pelkum J, Radice A, Norman GL, López Hoyos M, Lakos G, Buchner C, et al. Novel clinical and diagnostic aspects of antineutrophil cytoplasmic antibodies. J Immunol Res (2014) 2014:185416. doi:10.1155/2014/185416

9. Hellmich B, Csernok E, Fredenhagen G, Gross WL. A novel high sensitivity ELISA for detection of antineutrophil cytoplasm antibodies against proteinase3. Clin Exp Rheumatol (2007) 25(1 Suppl 44):S1-5.

10. Kain R. L29. Relevance of anti-LAMP-2 in vasculitis: why the controversy. Presse Med (2013) 42:584-8. doi:10.1016/j.lpm.2013.01.029

11. Ramos-Casals M, Stone JH, Cid MC, Bosch X. The cryoglobulinaemias. Lancet (2012) 379:348-60. doi:10.1016/S0140-6736(11)60242-0
12. Retamozo S, Brito-Zerón P, Bosch X, Stone JH, Ramos-Casals M. Cryoglobulinemic disease. Oncology (Williston Park) (2013) 27:1098-105.

13. Greco A, Rizzo MI, De Virgilio A, Gallo A, Fusconi M, Pagliuca G, et al. Goodpasture's syndrome: a clinical update. Autoimmun Rev (2015) 14:246-53. doi:10.1016/j.autrev.2014.11.006

14. Guilpain P, Mouthon L. Antiendothelial cells autoantibodies in vasculitisassociated systemic diseases. Clin Rev Allergy Immunol (2008) 35:59-65. doi:10. 1007/s12016-007-8069-3

15. Große K, Witte T, Moosig F, Hoyer BF, Lansche C, Schmidt RE, et al. Association of ferritin antibodies with Takayasu arteritis. Clin Rheumatol (2014) 33:1523-6. doi:10.1007/s10067-014-2764-2

Conflict of Interest Statement: The author declares that the research was conducted in the absence of any commercial or financial relationships that could be construed as a potential conflict of interest.

Copyright (C) 2015 Silva de Souza. This is an open-access article distributed under the terms of the Creative Commons Attribution License (CC BY). The use, distribution or reproduction in other forums is permitted, provided the original author(s) or licensor are credited and that the original publication in this journal is cited, in accordance with accepted academic practice. No use, distribution or reproduction is permitted which does not comply with these terms. 